\title{
Explanation of the Concept of Dark Energy and Its Role in Acceleratedexpansion of Universe
}

\author{
*Rishabh Mishra \\ School Of Naval Architecture and Ocean Engineering Indian Maritime University India \\ Corresponding Author: Rishabh Mishra
}

\begin{abstract}
This paper is all about explaining the role of dark energy in the accelerated expansion of universe by using simple mathematical tools. The aim of this paper is to render the complicated idea of expansion of universe in simple mathematics so that the people with less profound mathematical foundation can also understand this concept.
\end{abstract}

\section{Introduction}

Humans are curious and the quest to define the universe has always been the biggest challenge since the ancient times leading from the days of ancient INDIANS and GREEKS to the modern astrophysical sciences. And one question i.e. weather universe is in some constant shape or it is contracting or it is expanding, has always been the topper in the chronology of the priorities of this endless pursuit of cosmology. The next few sections will try to answer these questions by using simple mathematical forms, laws of mechanics and thermodynamics.

\section{SECTION:I \\ THE RUBBER BAND}

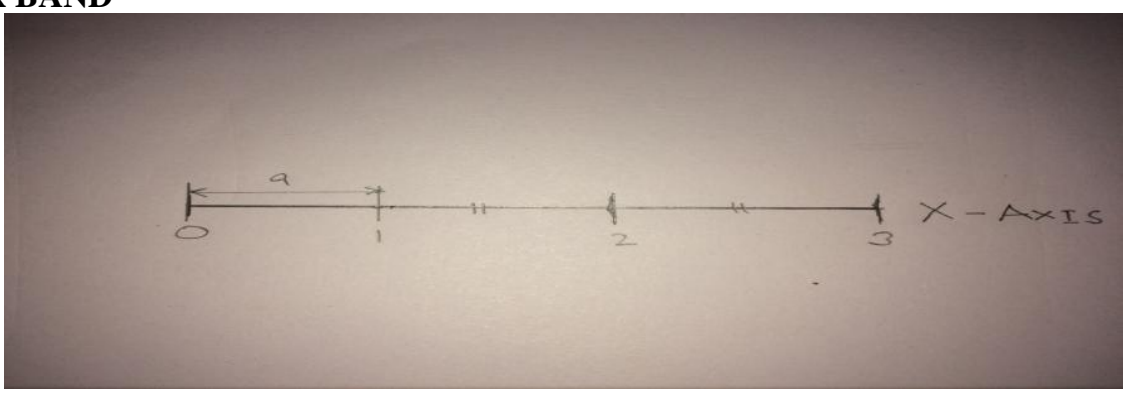

Let's start with a simple example of a rubber band. I have chosen the example of rubber band because I want to start with a single dimensional case. Pin one side of rubber band and straighten the rubber band as much as possible. Treat pin up point as the origin of this axis (rubber band). Mark four equidistant points on this rubber band. Now stretch it from one end. You will observe that the points are fixed at their positions but the distance between themhas increased. This is exactly what happens in case of universe. The galaxies are not moving from their position it $s$ the space-time fabric (on which the galaxies are lying) which is expanding in somewhat similar fashion as we have observed in case of rubber band.

Now let's see some mathematics over above qualitative analysis.

As the gap between any two coordinate points is increasing with time so take this as a function of time i.e. "a(t)".

Let's say initially this gap was "a".

Then the distance between any two points can be written as

$D=\Delta x a(t) \quad \ldots(1)$

$\frac{d D}{d t}=D^{\prime}=\Delta x a^{\prime}(t) \ldots 2$

$v=\Delta x a^{\prime}(t) \times \frac{a}{a} \quad \ldots 3$ 
$v=\frac{(\Delta x \times a) a^{\prime}(t)}{a}$

By using ...1 we can write

$v=\frac{D a}{a}=H D \ldots 4$

Where " $H$ " is known as HUBBLE'S CONSTANT.

The definition of velocity given above will play a very prominent role in our further discussions.

\section{SECTION: 2}

\section{THE ROLE OF GRAVITY (MATTER DOMINATED UNIVERSE)}

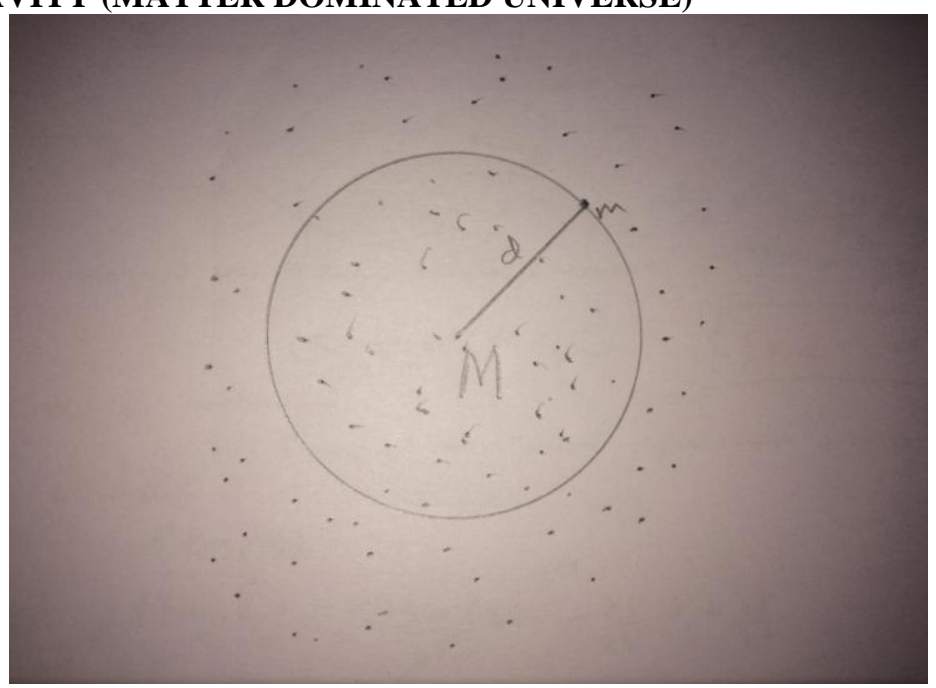

The dots shown in above figure are actually the galaxies. As we know there is no special point in universe ( because we consider universe to be homogenous and isotropic) but for our own calculations and to observe the effect of gravity let's specify a reference point and name it as ' $O$ '. Now take a galaxy at a distance ' $\mathrm{D}$ ' and lets assume at that place the space time is expanding at the rate " $v$ ". By the idea of Gauss we can say the effective gravitational force that is acting on the galaxy ' $\mathrm{m}$ ' is due only due to the mass of galaxies together i.e. "M".

But we know that

$v=H D$

$F=G \times \frac{M m}{D^{2}} \ldots 5$

$P E=-G \times \frac{M m}{D}$

$\frac{1}{2} m v^{2}-G \times \frac{M m}{D}=k$,

$v^{2}-\frac{2 G M}{D}=k^{\prime} \ldots \ldots \ldots .\left[k^{\prime \prime} / m=k^{\prime}\right]$

By using equation 1 and 2

$\Delta x^{2} a^{\prime}(t)-\frac{2 G M}{\Delta x a(t)}=k^{\prime} \ldots 6$

BUT

$M=V O L \times D E N S I T Y$

$M=\frac{4}{3} \pi D^{3} \times \rho(t)$ 
Substitute $M$ above equation we get

$\Delta x^{2} a^{\prime}(t)-\frac{8 \pi G}{3} a(t)^{2} \Delta x^{2} \rho(t)=k^{\prime}$

Divide throughout by $\Delta x^{2}$

$a^{\prime}(t)-\frac{8 \pi G}{3} a(t)^{2} \rho(t)=k$

SO,

$\frac{a^{\prime}(t)}{a(t)}=\frac{8 \pi G}{3} \rho(t)-\frac{k}{a(t)^{2}} \ldots 7$

Depending upon the value of ' $k$ ' following conclusions can be made

$\mathrm{k}>0$ closed universe

$\mathrm{k}<0$ open universe

$\mathrm{k}=0 \quad$ flat universe

Let us take a cube of side ' $a$ ' (which is actually, according to our previous discussions is the distance between two points in coordinate axes.)

$\frac{a^{\prime}(t)^{2}}{a(t)^{2}}=\frac{8 \pi G \rho(t)}{3}-\frac{k}{a(t)^{2}}$

$$
\rho(t)=\frac{M}{a(t)^{3}}
$$

According to observations without the inclusion of the concept of dark energy the universe seems out to be a flat universe hence we take the value of ' $k$ ' to be zero.

$k=0$

Hence,

$\frac{a^{\prime}(t)^{2}}{a(t)^{2}}=\frac{8 \pi G \rho(t)}{3} \ldots 8$

Substitute the value of density

$\frac{a^{\prime}(t)}{a(t)}=\frac{8 \pi G}{3}\left(\frac{M}{a(t)^{3}}\right)$

Let's assume

$a(t)=c t^{p}$

$a^{\prime}(t)=p t^{p-1}$

Divide above two equations to each other

$\frac{a^{\prime}(t)}{a(t)}=\frac{p}{t}$

So,

$\frac{p^{2}}{t^{2}}=\frac{8 \pi G}{3}\left(\frac{M}{a(t)^{3}}\right)$

$\frac{p^{2}}{t^{2}}=\frac{8 \pi G}{3}\left(\frac{M}{c^{3} t^{3 p}}\right)$

By comparing above two equations we can write,

$t^{2}=t^{3 p}$ 
We get,

$p=\frac{2}{3}$

$a=c t^{\frac{2}{3}} \ldots 9$

\section{SECTION: 3 RADIATION DOMINATED UNIVERSE}

In the previous section we discussed everything related to matter. But early universe was not composed of matter it was composed of high energy-radiations.

So let's have a look over radiations

Again take that cube but in this case it is not filled with matter instead it is filled with QUANTAS of radiation.

Let's take a single quanta and analyse it

$E=h v=h c / \lambda(t)$

$\lambda$ is time dependent because with the expansion of space-time the wavelength should also increase. Because of which the high energy radiations present at that time are now in 'microwave region'.

WHICH IS POPULARLY KNOWN AS CMBR i.e. "COSMIC MICROWAVE BACKGROUND RADIATION".

Which acts like the carbon footprint of universe.

We know that

$E=m c^{2}$

And ,

$E \propto \frac{1}{\lambda(t)} \propto \frac{1}{a(t)}$

$\mathrm{SO}$,

$$
E=\frac{N}{a(t)}
$$

We can write,

$$
m c^{2}=\frac{N}{a(t)}
$$

Mass density of this system can be writtenas,

$\rho(t)=\frac{N}{c^{2} a(t)^{4}} \ldots 10$

Now put the relation ' 10 ' in equation ' 7 '

We get,

$\frac{a^{\prime}(t)^{2}}{a(t)^{2}}=\frac{8 \pi G}{3}\left(\frac{N}{a(t)^{4}}\right)$

But,

$a=c t^{p}$

$\frac{a^{\prime}(t)^{2}}{a(t)}=\frac{p^{2}}{t^{2}}=\frac{8 \pi G}{3}\left(\frac{N}{c^{4} t^{4 p}}\right)$

$t^{2}=t^{4 p}$

So, 
$p=\frac{1}{2}$

$a=c t^{\frac{1}{2}} \ldots 11$

\section{SECTION: 3 DARK ENERGY DOMINATED UNIVERSE}

It was observed by Hubble that the universe was actually expanding at an accelerated rate. The following mathematical structure can explain it very easily.

Let us say that,

$$
\rho(t)=\frac{c}{a^{3(w+1)}}
$$

Where ' $c$ ' is some constant

Where take,

$$
\begin{gathered}
\begin{array}{c}
w=0, \text { for matter dominated universe } \\
\quad w=\left(\frac{1}{3}\right) \text {, for radiation dominated universe } \\
w=-1 \text {, for dark energy dominated universe }
\end{array}
\end{gathered}
$$

So for dark energy dominated universe, equation 7 becomes following,

$\frac{a^{\prime}(t)^{2}}{a(t)^{2}}=\frac{8 \pi G}{3} \rho$

$a^{\prime}(t)=a(t) \sqrt[2]{\frac{8 \pi G \rho}{3}}$

If we solve the above differential equation,

We get the following solution,

$a(t)=c e^{\sqrt{\frac{8 \pi G \rho}{3}}(t)} \ldots 12$

\section{SECTION: 4}

\section{Conclusion}

If we look at equation no ' 12 ' the dimensional expansion of universe is exponentially related to time which itself shows the accelerated expansion of universe. This concept could be explained only and only if we take dark energy into consideration. Hence it proves the existence of dark energy.

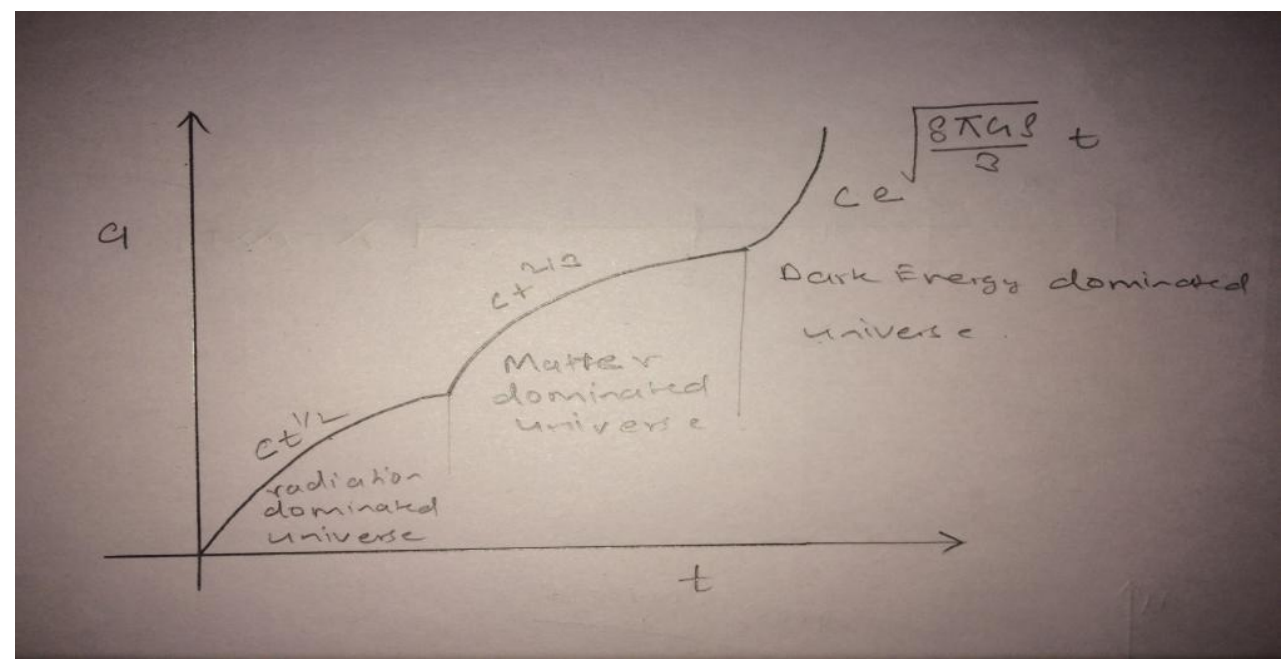

The above graph shows the different phases of expansion of universe. 


\section{References}

[1] Lectures of Dr. Physics A.

[2] Space-time and geometry (An introduction to General Relativity) by Sean M. Carroll.

IOSR Journal of Applied Physics (IOSR-JAP) is UGC approved Journal with Sl. No. 5010, Journal no. 49054.

Rishabh Mishra. "Explanation of the Concept of Dark Energy and Its Role in Acceleratedexpansion of Universe." IOSR Journal of Applied Physics (IOSR-JAP) 9.4 (2017): 01-06. 